\title{
Creating a Dynamic Map Template for National Scale Malaria Treatment Planning in Zambia
}

\author{
Matthew Heaton \\ Center for International Earth Science Information Network (CIESIN), Columbia University, mheaton@ciesin.columbia.edu
}

Keywords: Atlas, Participatory mapping, Public health, Tropical diseases

\begin{abstract}
:
The national government of Zambia aims to achieve 100 percent malaria vector control coverage nationwide via indoor residual spray (IRS) and insecticide-treated nets (ITN) distribution by the end of 2021. In order to meet this goal, health professionals require an inventory of (1) the spatial distribution of settled areas to treat, (2) the number of residential buildings to allocate resources for, and (3) harmonised district boundaries subdividing treatment areas. These critical components were introduced to a technical audience by the production of a national-scale atlas of Zambia. Through a collaborative effort by GRID3 (Geo-Referenced Infrastructure and Demographic Data for Development), Akros, and other Zambia National Malaria Elimination Programme partners, this atlas was designed, printed at scale, and supplied to health professionals in a series of treatment campaign planning sessions.
\end{abstract}

Three core criteria drove atlas map design: at-a-glance discernment of the spatial distribution and intensity of settlements; maintaining legibility of map content when subdividing the full extent of Zambia into regularlyproportioned index grid cells; and map reproducibility or interoperability at scale for future rounds of vector control campaigns. The country of Zambia was platted into a series of nested grids to drive 289 atlas page extents at manageable scales and to generate zonal statistics to guide participatory pen-and-paper planning sessions. Map templates first used in 2020 are currently being updated following feedback and repurposed for 2021 national treatment sessions.

The potential outcomes of this intervention include: ensuring that all settlements within a district are accounted for in service delivery; ensuring that there are enough vector control resources to reach all settlements across all districts; improving understanding of the accuracy of reported coverage from previous years; and communicating and harmonising geospatial data across the government and its partners.

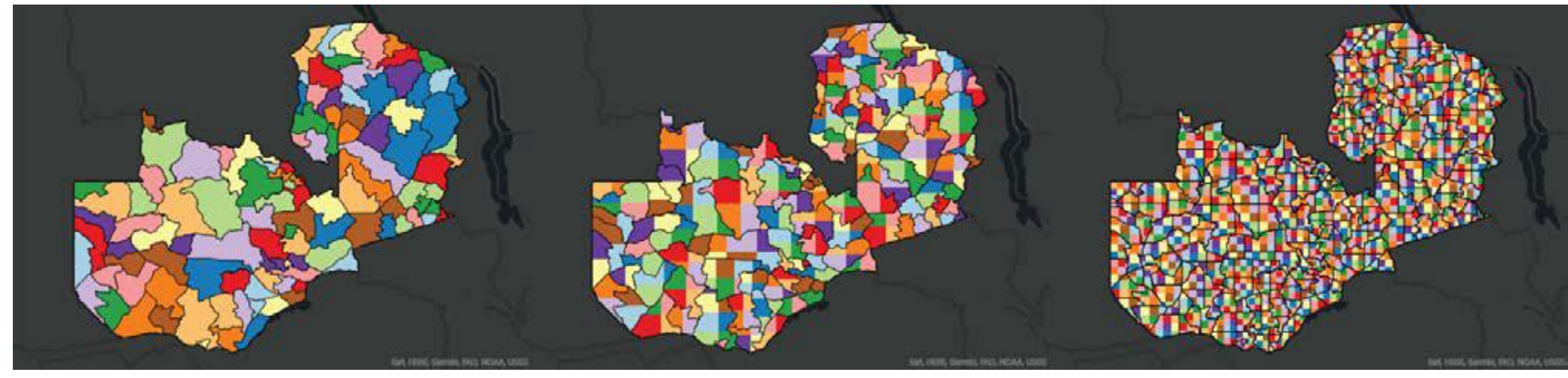

Figure 1. A flexible grid system was devised using harmonised district boundaries (left) to generate the atlas page index (center) and nested reference grid cells (right). Reference grids were used to summarize residential building and population counts, and to create corresponding alphanumeric settlement names for organizational purposes. 


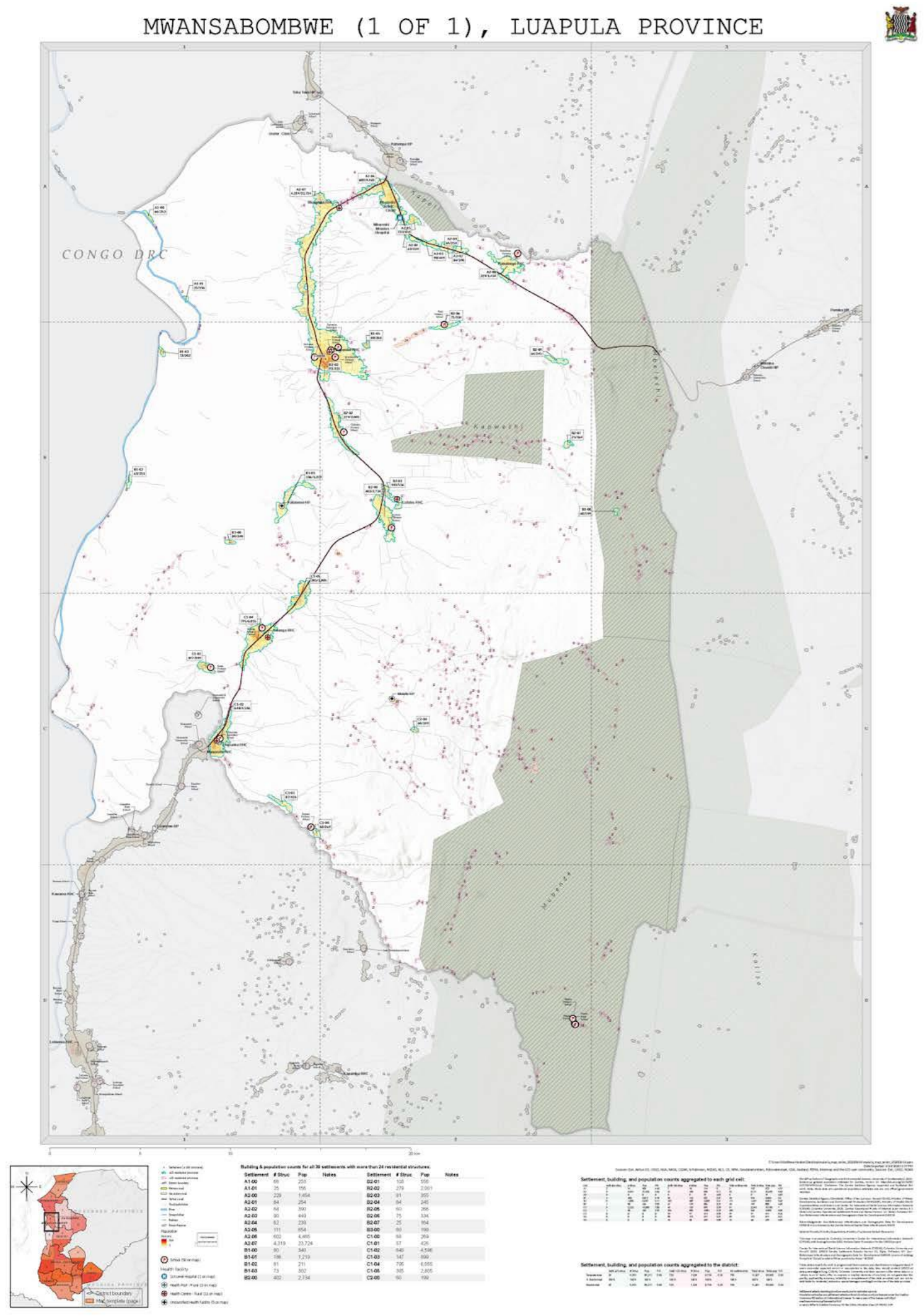

Figure 2. An example of a page created with the dynamic map template. 


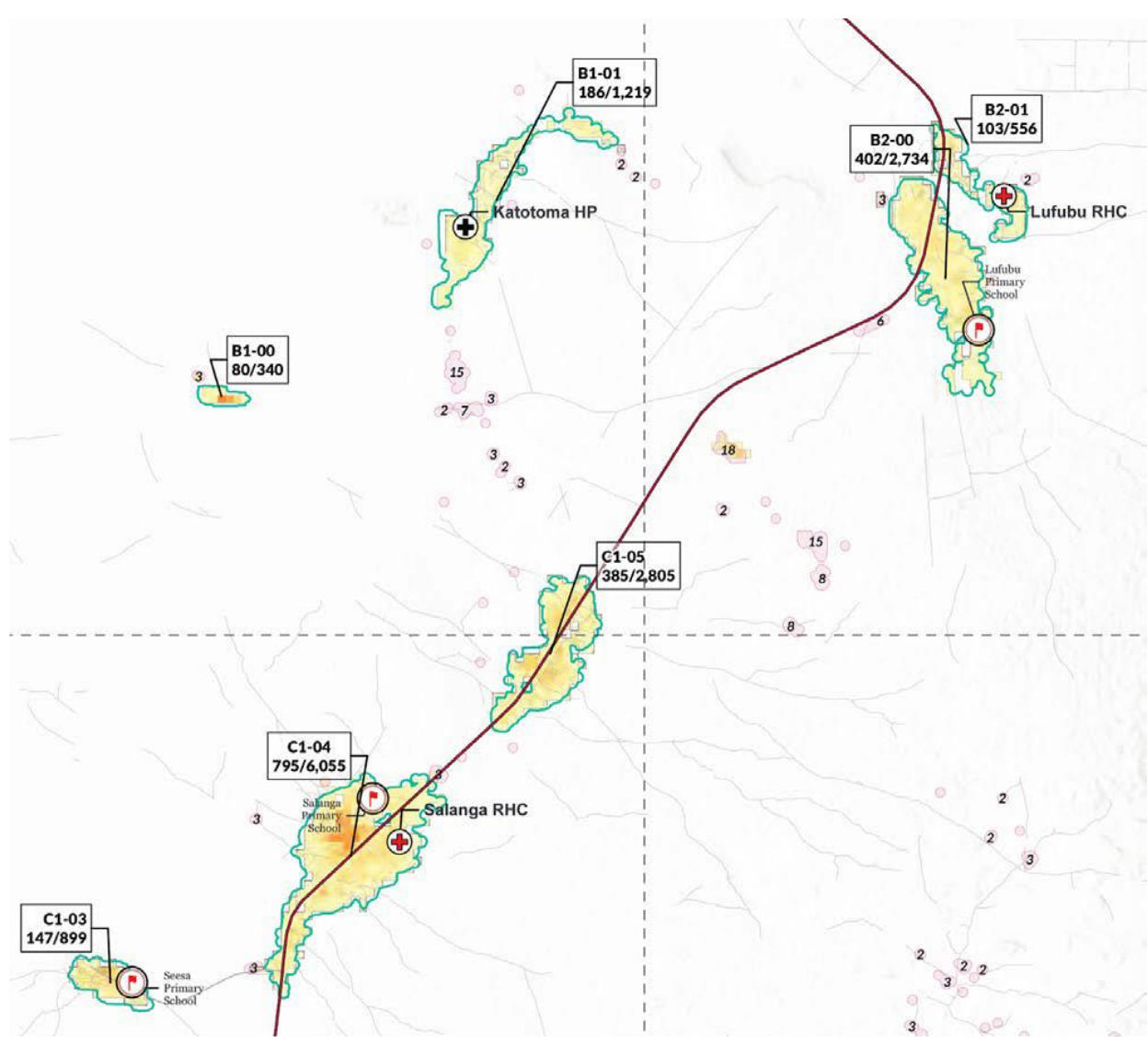

Figure 3. Map detail. Magenta hamlets are targeted with ITN treatment; teal-outlined settlement areas are targeted with IRS. Settlements are organized into human-readable alphanumeric grid cells, and contain information on residential building count and total estimated population
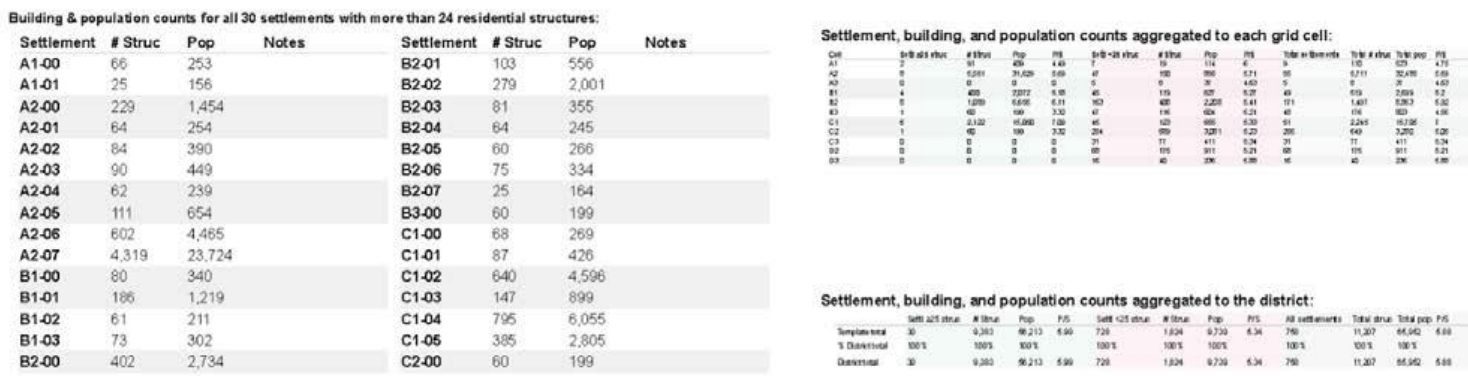

Figure 4. Summary statistics detail. Tables are updated dynamically based on each map page's extent. 


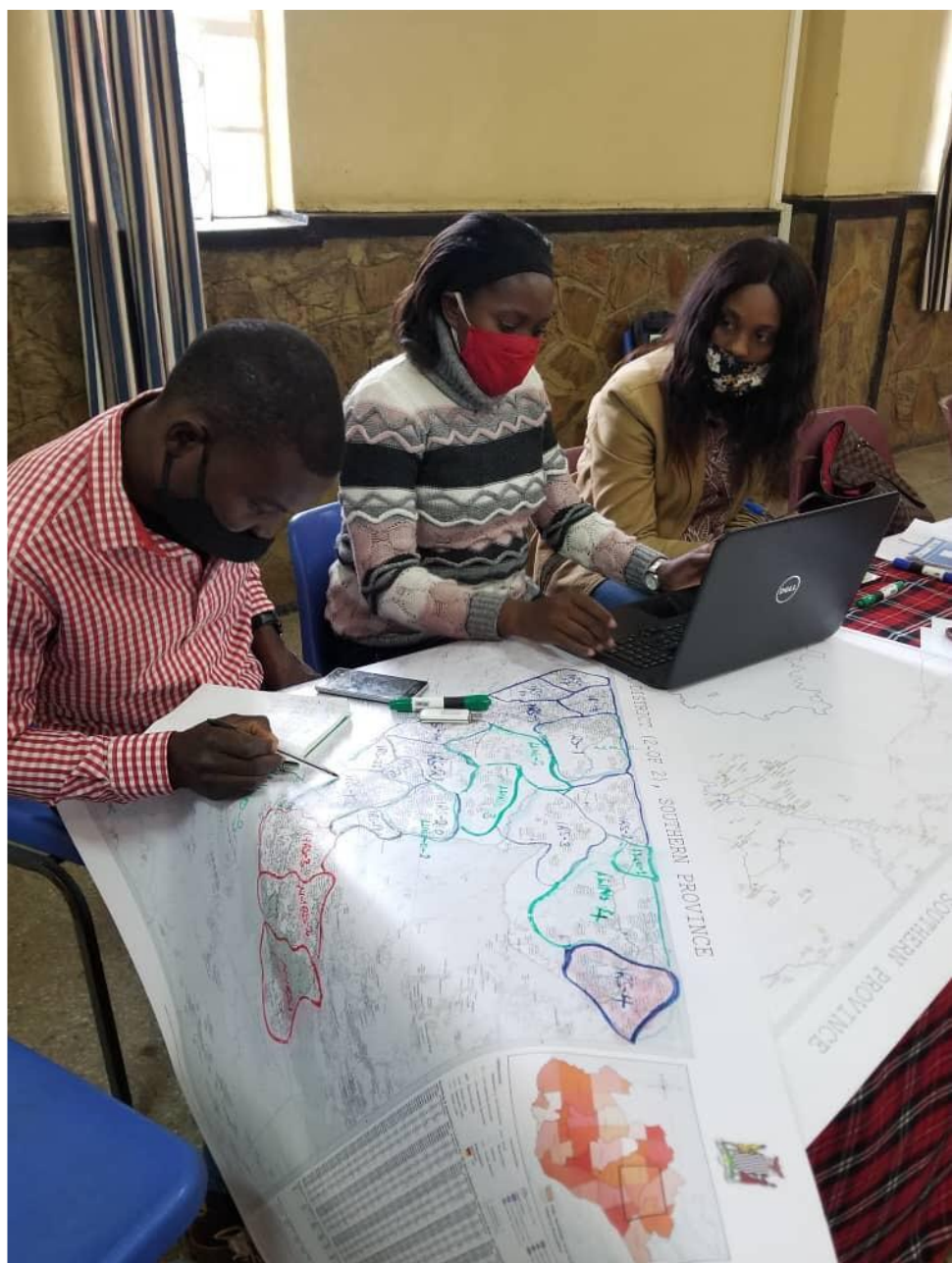

Figure 5. Maps in use during a treatment planning session, July 2020. 